\title{
Effect of normal stresses on the results of thermoplastic mold filling simulation
}

\author{
Alexander Bakharev, David Astbury, Shishir Ray, Franco S. Costa and Russell Speight \\ ${ }^{1}$ Autodesk, 259-261 Colchester Rd., Kilsyth, Victoria 3137, Australia
}

\begin{abstract}
The paper deals with the effect of the normal stresses on the predicted flow front during the filling stage of thermoplastic injection molding. The normal stresses are predicted using the non-linear Criminale-Ericksen-Filbey model (a variant of the second-order fluid rheological model with viscosity, first and second normal stress coefficients dependent upon magnitude of shear rate) incorporated into a comprehensive 3D simulation software for mold-filling analysis. The additional stress term allows the prediction of the so called ear-flow effect (melt racing on the edges of the cavity).

Keywords: Finite Elements, Injection Molding, Simulation, Rheology, Normal stress difference, Ear-flow.
\end{abstract}

\section{Introduction}

Thermoplastic injection molding is the most common manufacturing process for producing plastic parts. Material is fed into a heated barrel, mixed, and forced into a mold cavity where it cools and hardens to the configuration of the cavity. Significant progress has been achieved in three dimensional finite element simulation of plastic filling the mold (mold filling analysis) [1]. Typically in commercial simulations polymer melt is considered as generalized Newtonian fluid, there the deviatoric stress tensor is proportional to the deviatoric deformation rate, the scalar coefficient connecting the shear rate and shear stress, known as viscosity, is dependent upon temperature, shear rate invariants, pressure and other factors [2]. However, the generalized Newtonian fluid model does not predict any normal stress differences during simple shear flow whereas real polymers usually exhibit significant normal stress differences.

In this current work, we develop a finite element mold-filling program that allows incorporation of the Criminale-Ericksen-Filbey viscoelastic model that can accurately predict normal stress differences in a wide range of temperatures and shear rates [3]. One of the motivators for this development was potentially improving the prediction of ear-flow, a little understood phenomenon of the more rapid advance of the flow front on the edge of a mold cavity than in the center of the cavity [4].

\footnotetext{
${ }^{\mathrm{a}}$ Corresponding author: Alex.Bakharev@autodesk.com
}

\section{Ear-Flow Phenomenon}

Numerous cases have been observed in industrial injection molding practice of amorphous materials which exhibit a race-track or ear-flow effect. This is the more rapid advance of the flow front at the edges of the molding cavity than in the center of the cavity, usually observed at elevated injection speed. This flow-leading effect at the edge cannot be explained by differences in cavity wall section thickness. In the worst cases, this race-tracking leads to air-traps and visual defects in the molded part. A typical flow front propagation demonstrating the ear-flow in a polystyrene material is shown on Figure 1.

As was originally suggested by experimental observations of Murata et al. [5], and confirmed by moldfilling simulations of Costa et al. [4], in many cases the ear flow is caused by the higher polymer temperatures in the edge region. The temperature rise is caused by shear heating in the high-shear region (close to the boundary) of the runners and gates. This temperature rise is then convected into the cavity, favoring the cavity edge due to the distribution pattern in the gate. The effect of the preferential convection of the temperature rise from the shear regions of the runners is also similar to the development of flow imbalance in geometrically balanced feed systems explained by Cook et al. [6].

However in many practical injection molding cases ear flow phenomenon is observed for conditions where very little shear heating is predicted, thus, rising suspicions that there is also another mechanism responsible for the ear flow effect. Since normal stress differences caused by in-plane shear near the cavity edges may push polymer perpendicular to the flow they are a 
candidate for such a mechanism. Our simulation was used to test this possibility.

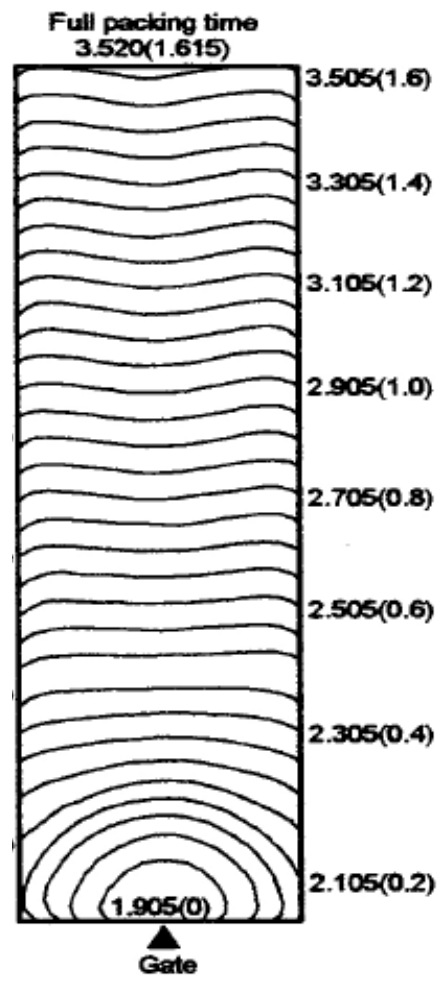

Figure 1 Flow front shapes obtained by Murata et al. [5] for a Polystyrene material in a glass insert mold.

\section{Mathematical model}

Filling stages of injection molding process are described by the combination of the momentum equation 1 :

$$
\frac{\partial}{\partial t}(\rho \vec{v})=\rho \vec{g}+(\nabla \cdot \underline{\sigma})+\nabla p-(\nabla \cdot(\rho \vec{v} \vec{v}))
$$

mass continuity (equation 2 ):

$$
\frac{\partial \rho}{\partial t}+\nabla \cdot(\rho \vec{v})=0
$$

and the energy equation (3):

$$
\begin{aligned}
& \rho c_{p}\left(\frac{\partial T}{\partial t}+\vec{v} \cdot \nabla T\right)= \\
& -\frac{1}{\rho}\left(\frac{\partial \rho}{\partial T}\right)_{p} T\left(\frac{\partial p}{\partial t}+\vec{v} \cdot \nabla p\right) \\
& +p \nabla \cdot \vec{v} \\
& +(\underline{\sigma: \nabla \vec{v}}) \\
& +\nabla \cdot(k \nabla T)
\end{aligned}
$$

together with appropriate material equations and boundary conditions described in [1] and [2]. In the simplest mold filling case the material equations include generalized Newtonian rheological equation:

$$
\underline{\sigma}=2 \eta(T, \dot{\gamma}, p) \underline{D}
$$

and density or PVT equation:

$$
\rho=\rho(p, T)
$$

Boundary conditions are set on the mold-plastic interface, on the melt flow front and on the injection surfaces.

On the mold-plastic interface the boundary conditions are set as:

$$
\begin{aligned}
& T=T_{\text {mold }} \\
& \vec{v}=\overrightarrow{0}
\end{aligned}
$$

On the melt flow front $(F)$ the boundary conditions are set as

$$
\begin{aligned}
& \nabla T \bullet \vec{n}_{F}=0 \\
& \underline{p+\sigma \bullet \vec{n}_{F}=0}
\end{aligned}
$$

where $\vec{n}_{F}$ is the normal to the flow front $F$.

On the injection surface $(I)$ the boundary conditions are set as

$$
\begin{aligned}
& T=T_{\text {melt }} \\
& \frac{p+\sigma \bullet \vec{n}_{I}=\text { const }}{\iint_{I} \vec{v} \bullet \vec{n}_{I} d I=Q(t)}
\end{aligned}
$$

where $\vec{n}_{I}$ is the normal to the injection surface $I$ and $Q(t)$ is the injection flow rate.

The system of equations (1)-(12) is solved using a specialized finite element method customized for the typical conditions of the injection molding process described in [1].

In order to incorporate the effect of normal stresses we implemented material rheological function connecting stresses $(\sigma)$ with flow conditions that follows CriminaleEricksen-Filbey model [3]:

$$
\begin{aligned}
& \underline{\sigma}=2 \eta(T, \dot{\gamma}, p) \underline{D} \\
& -\psi_{1}(T, \dot{\gamma}, p) \underline{D}+4 \psi_{2}(T, \dot{\gamma}, p) \underline{D} \bullet \underline{D}
\end{aligned}
$$

In the equation (4) $\underline{\sigma}$ is the deviatoric stress tensor; $\underline{D}$ is the deformation rate tensor, $\eta(T, \dot{\gamma}, p)$ is viscosity dependent upon temperature $T$, scalar shear rate $\dot{\gamma}=\|\underline{D}\|$ and pressure $p ; \underline{D}$ denotes the upper convective derivative of the deformation rate tensor:

$$
\stackrel{\nabla}{D}=\frac{\partial}{\partial t} \underline{D}+\vec{v} \bullet \nabla \underline{D}-(\nabla \vec{v})^{T} \bullet \underline{D}-\underline{D} \bullet \nabla \vec{v}
$$

In equation (14), $\vec{v}$ is velocity and functions $\psi_{1}(T, \dot{\gamma}, p)$ and $\psi_{2}(T, \dot{\gamma}, p)$ are correspondingly the first and the second normal stress difference functions. Equation (13) is an extension of the generalized Newtonian equation (4). 
Following established practice for mold-filling simulation we use Cross-WLF model [2] for the viscosity model:

$$
\eta(T, \dot{\gamma}, p)=\frac{\eta_{0}(T, p)}{1+\left(\frac{\eta_{0}(T, p) \dot{\gamma}}{\tau^{*}}\right)^{1-n}}
$$

Where

$$
\eta_{0}(T, p)=D_{1} \exp \left[-\frac{A_{1}\left(T-T^{*}\right)}{A_{2}+\left(T-T^{*}\right)}\right],
$$

$T^{*}$ is the glass transition temperature, $A_{2}=A_{3}+D_{3} p$

$D_{1}, A_{1}, A_{3}, D_{3}$ and $n$ are empirical coefficients. Autodesk Moldflow material library stores quite an extensive data of the Cross-WLF parameters for thousands of material grades.

To evaluate functions $\psi_{1}(T, \dot{\gamma}, p)$ and $\psi_{2}(T, \dot{\gamma}, p)$ when viscosity is known we followed the so called CoxMertz Abnormal Rule as described by V. Sharma and G.H. McKinley [7].

$$
\psi_{1}(T, \dot{\gamma}, p) \approx 2 \dot{\gamma}^{-1} \eta\left(1-\frac{\eta_{c}^{2}}{\eta^{2}}\right)^{0.5}\left(\frac{\eta_{c}^{2}}{\eta^{2}}\right)^{0.7}
$$

In the equation $7, \eta=\eta(T, \dot{\gamma}, p)$ is the steady state viscosity and $\eta_{c}=\frac{\partial}{\partial \dot{\gamma}}(\eta(T, \dot{\gamma}, p) \dot{\gamma})$ is the tangential or consistency viscosity. As shown in [7] equation 7 allows relatively accurate estimation of the first normal stress difference function if Cross-WLF parameters are known.

Finally, to estimate the second normal stress difference function $\psi_{2}(T, \dot{\gamma}, p)$ we assume that it is proportional to the first normal stress difference function:

$$
\psi_{2}(T, \dot{\gamma}, p)=K_{\psi} \psi_{1}(T, \dot{\gamma}, p)
$$

where the coefficient $K_{\psi}$ takes values between -0.5 and 0 [3].

The set of equations 1-17 was integrated into a special build of 3D flow solver of Autodesk Moldflow Insight 2017 software and the resulting program was used for simulation of injection molding processes. The addition included calculation of the additional normal stress tensor field:

$$
\underline{\sigma_{n}}=-\psi_{1}(T, \dot{\gamma}, p) \underline{\nabla} \underline{D}+4 \psi_{2}(T, \dot{\gamma}, p) \underline{D} \bullet \underline{D}
$$

using equations (15)-(18). Then we apply additional nodal forces $\vec{N}_{i}$ to each of the filled nodes $i$ calculated as:

$$
\vec{N}_{i}=\oiint_{S_{i}} \underline{\sigma_{n}} \bullet \vec{n}_{S_{i}} d S_{i}
$$

where $S_{i}$ is the surface of the control volume of the node $i$. At the each time step the algorithm iterated the velocity-pressure solver together with the calculations of the normal stress by equations (18) and (19) until they converge. The rest of the mold filling simulation algorithm described in [1] was left intact.

\section{Filling of a thin rectangular cavity}

\subsection{Case study}

In order to estimate the effect of normal stresses on flow front propagation we use a filling simulation of a simple thin rectangular plaque of $100 \mathrm{~mm}$ in length, $20 \mathrm{~mm}$ in width and $2 \mathrm{~mm}$ in thickness shown on Figure 2. The plague being filled by the generic polymethylmethacrylate (PMMA) material. The filling time is 1 second. The cavity is meshed by 4 node tetrahedral elements with at least 10 layers of elements through the thickness. Melt inlet boundary conditions are applied along one of the short edges of the plaques in the style of a film or fan gate.

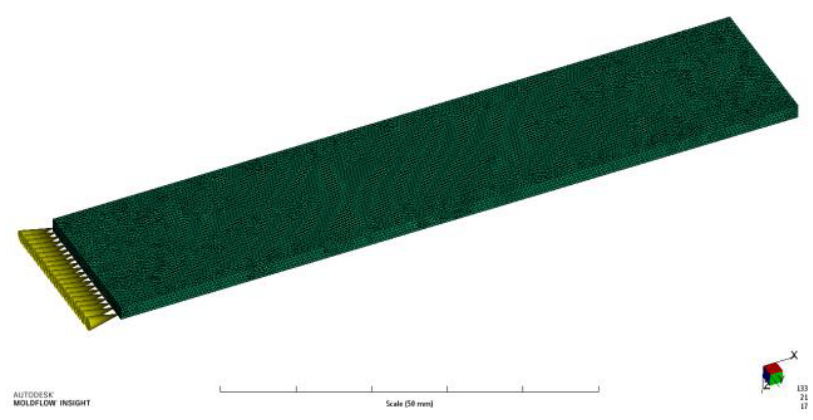

Figure 2 Illustrative molding case

Three rheological models were considered:

- No normal stresses

- First normal stress difference estimated from the Cox-Mertz Abnormal Rule but no Second normal stress differences: coefficient $K_{\psi}=0$

- First normal stress difference estimated from the Cox-Mertz Abnormal Rule and large Second normal stress differences $K_{\psi}=-0.5$.

All other rheological, thermal and mechanical material parameters were taken from the standard material library.

All three cases simulation do not show any significant shear heating in the edge area (see Figure 3) as was expected because the runners and gates were not modeled. 


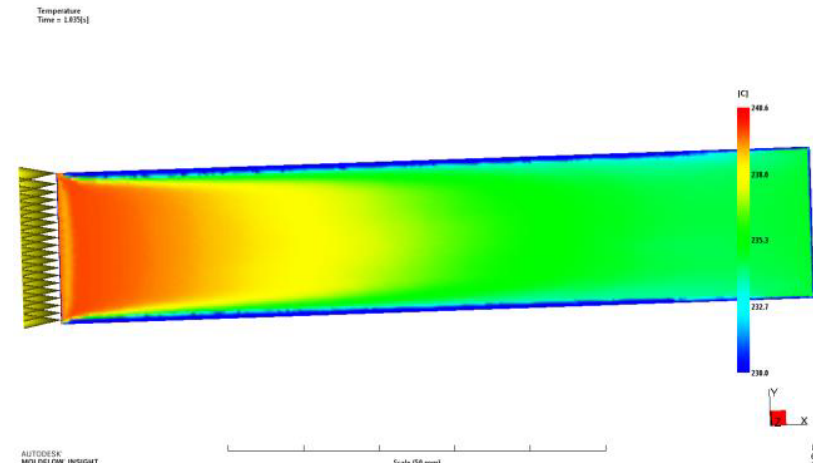

Figure 3 Temperature distribution at the end of fill. No normal stresses case. The cutting plane is in the middlethickness plane of the cavity.

\subsection{No normal stresses case}

The simulation without normal stresses does not predict any ear flow phenomenon as shown in Figure 4.

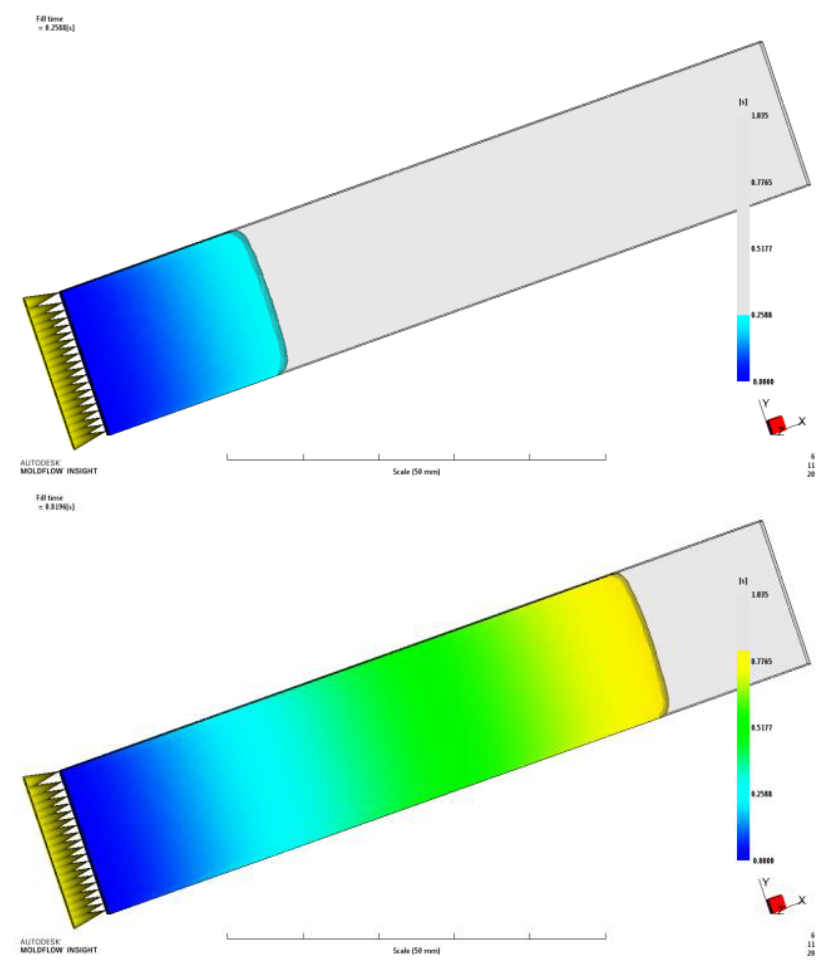

Figure 4 Flow front positions for the case with no normal stress.

\subsection{First normal stress differences only}

When only the first normal stress difference is included, the flow front propagation pattern is very similar to the case of no normal stress. As shown in Figure 5 there is no ear flow phenomenon in this case either.

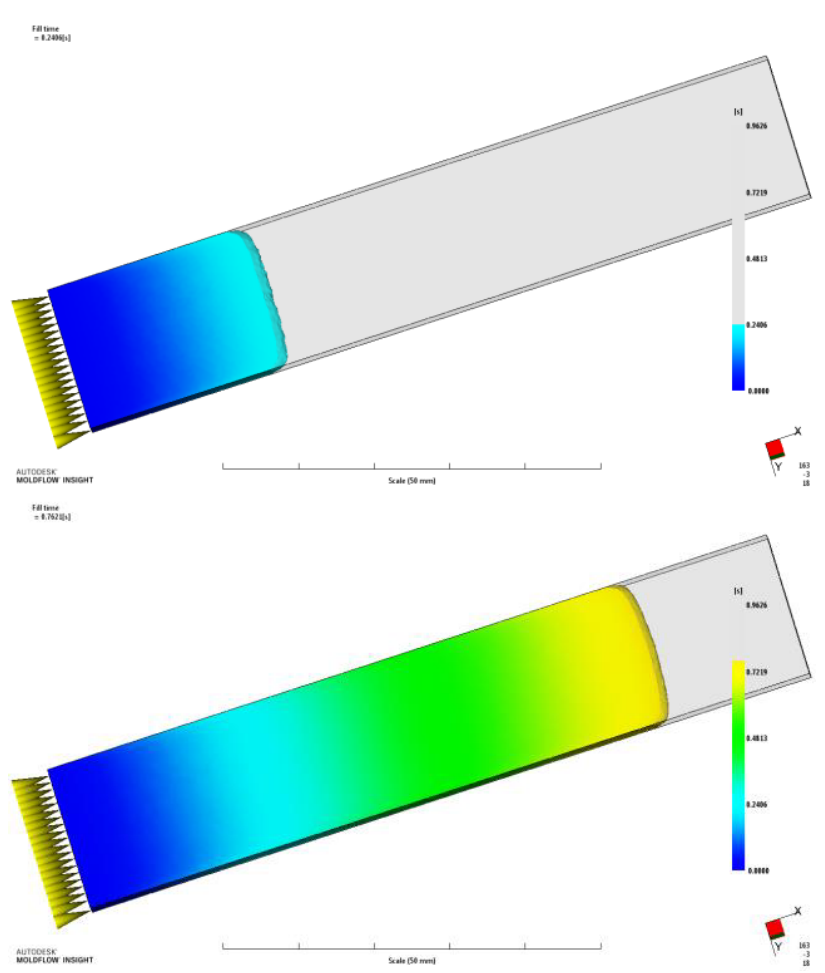

Figure 5 Flow front positions for the case with the first normal stress differences only.

\subsection{First normal differences and maximal second normal differences}

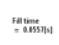

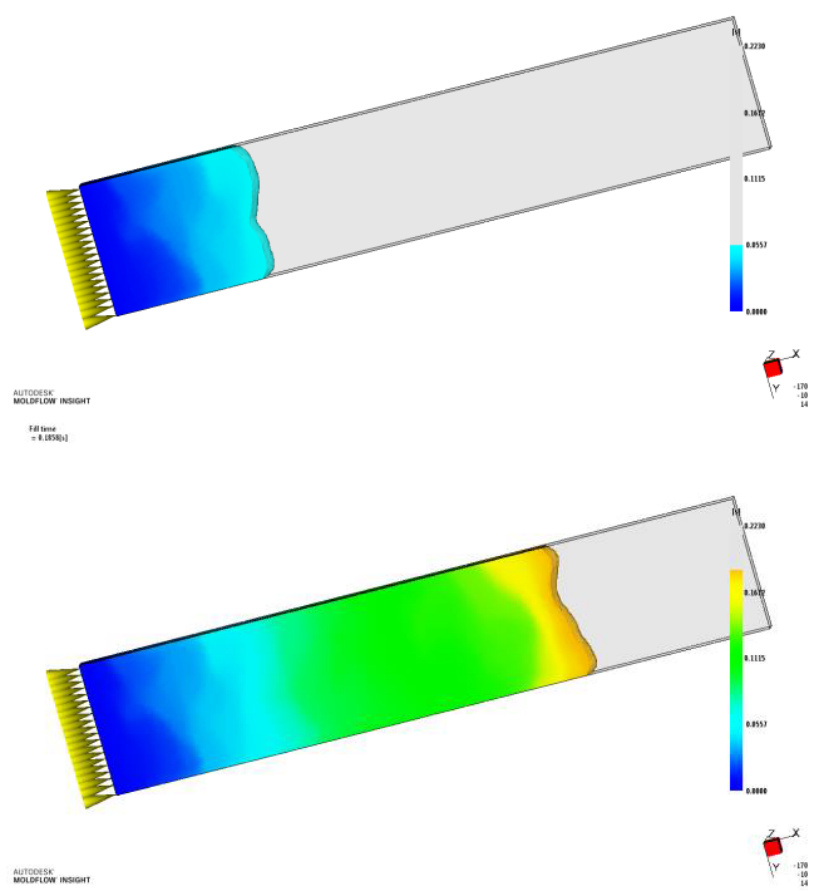

Figure 6 Flow front positions for the case with the first and second normal stress differences.

The flow front distribution from the simulation using first and second normal stress differences is shown in Figure 6. A significant ear flow effect can be seen. The effect is 
quite prominent despite a moderate magnitude of normal stresses up to $\sim 30 \mathrm{kPa}$ (see Figure 7).

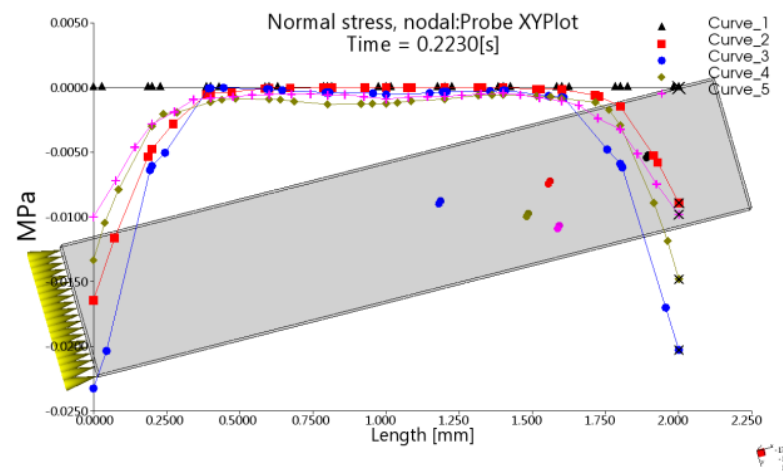

Figure 7 Normal stress difference $\sigma_{y y}-\sigma_{z z}$ component, thickness probe.

The results of these simulations show that the second normal stress difference can be an important contributing factor to the ear flow phenomenon.

\section{Conclusions}

An integrated system of 3D mold filling simulation that takes into account nonlinear rheological properties of normal stress differences is presented.

The second normal stress difference appears to be an important contributing factor to the ear flow phenomenon while the first normal stress difference does not affect much the flow front propagation.

\section{References}

1. Talwar, K., Costa, F., Rajupalem, V., Antanovski,
L and Friedl, C., Three Dimensional Simulation of Plastic Injection Molding. SPE ANTEC Proceedings (1998)

2. Kennedy, P. and Zheng, R., Flow Analysis of Injection Molds $2 d$ edition. Munich : Carl Hanser Verlag (2013)

3. Macosko, Christopher W. Rheology: principles, measurements and applications. New York: WilleyVCH, (1994)

4. Costa, F.S., Yokoi, H., Murata, Y. and Kennedy, P.K., Numerical Simulation of Ear-flow: The Faster Advance of the Flow Front at the Edge of a Cavity. Japan : Proc.22nd Annual Conference of the Polymer Processing Society (2006)

5. Murata, Y., Abe, S. and Yokoi, H., Experimental Analysis of Flow Front Advancing Phenomena. SeikeiKakou 15(10), pp.706-713 (2003)

6. Cook, P. S., Yu, H. Kietzmann, C. V., Costa, F. S., Prediction of Flow Imbalance in Geometrically Balanced Feed Systems. Boston: SPE ANTEC Proceedings, 2005.

7. Sharma, V. and McKinley, G.H., An Intriguing Empirical Rule for Computing the First Normal Stress Difference from Steady Shear Viscosity Data for Polymer Solutions and Melts. Rheol. Acta (2012). 